\title{
]jfis
}

\section{Multiple Instance Mamdani Fuzzy Inference}

Amine B. Khalifa and Hichem Frigui

CECS Department, University of Louisville, Louisville, KY, USA

\begin{abstract}
A novel fuzzy learning framework that employs fuzzy inference to solve the problem of Multiple Instance Learning (MIL) is presented. The framework introduces a new class of fuzzy inference systems called Multiple Instance Mamdani Fuzzy Inference Systems (MIMamdani). In multiple instance problems, the training data is ambiguously labeled. Instances are grouped into bags, labels of bags are known but not those of individual instances. MIL deals with learning a classifier at the bag level. Over the years, many solutions to this problem have been proposed. However, no MIL formulation employing fuzzy inference exists in the literature. Fuzzy logic is powerful at modeling knowledge uncertainty and measurements imprecision. It is one of the best frameworks to model vagueness. However, in addition to uncertainty and imprecision, there is a third vagueness concept that fuzzy logic does not address quiet well, yet. This vagueness concept is due to the ambiguity that arises when the data have multiple forms of expression, this is the case for multiple instance problems. In this paper, we introduce multiple instance fuzzy logic that enables fuzzy reasoning with bags of instances. Accordingly, a MI-Mamdani that extends the standard Mamdani inference system to compute with multiple instances is introduced. The proposed framework is tested and validated using a synthetic dataset suitable for MIL problems. Additionally, we apply the proposed multiple instance inference to fuse the output of multiple discrimination algorithms for the purpose of landmine detection using Ground Penetrating Radar.
\end{abstract}

Keywords: Fuzzy inference, Mamdani FIS, Multiple Instance Learning, Algorithm fusion

\section{Introduction}

Fuzzy inference is a powerful modeling framework that can handle computing with knowledge uncertainty and measurements imprecision effectively [1]. It is a process based on concepts from fuzzy set theory and fuzzy reasoning. It performs a non-linear mapping from an input space to an output space by deriving conclusions from a set of fuzzy if-then rules and known facts [2]. Fuzzy inference has been successfully applied to a wide range of problems, mainly in system modeling and control [3-5]. Most of the proposed fuzzy inference methods gained success because of their ability to leverage expert knowledge to identify the model parameters [6]. This practice simplifies system design and ensures that the knowledge base (if-then rules) used by the system is easy to interpret [7].

More recently, fuzzy inference has increasingly been applied to more advanced applications, such as content-based information retrieval [8], image segmentation [9], image annotation [10], pattern recognition [11], recommender systems [12], and multiple classifier fusion [13]. The aforementioned applications are more challenging as they require extensive knowledge base 
to accommodate for various scenarios. Since this diverse knowledge base cannot be fully provided by domain experts, datadriven techniques are typically used to identify and learn the fuzzy inference system's parameters [14, 15]. In this later technique, supervised and unsupervised learning algorithms are devised to learn the parameters of the fuzzy inference system (i.e. learn the knowledge base) from a set of labeled training data. For instance, a clustering algorithm (unsupervised learning) can be used to identify local contexts of the input space, and a linear classifier (supervised learning) can be used to learn decisions within each of the contexts. Thus, substituting the traditional expert knowledge based system's identification methods, with more scalable, adaptive, and broader learning methods.

Typically, in supervised learning problems, access to large labeled training datasets improves the performance of the devised algorithms by increasing their robustness and generalization capabilities. Nowadays, access to such large datasets is becoming more convenient. In fact, we generate about 2.5 quintillion bytes of data everyday ( $90 \%$ of the data in the world today has been created in the last two years alone) [16]. This data is continuously collected from sensors that measure environmental information, posts to social media sites such as Flickr [17], digital pictures and videos uploaded to advertisement websites such as Craigslist [18], etc. This trend is not expected to slow down anytime soon and is fueled by the drastic decrease in the cost of data storage. However, for a supervised leaning method to benefit from this data, it needs to be carefully preprocessed, filtered, and labeled. Unfortunately, this process can be too tedious as the vast portion of the collected data is unstructured, labeled ambiguously, and at a coarse level. For example, in image annotation, tags could be used as indicators of the existence of objects of interests within the images (sky, sea, beach,...). However, the exact location of those objects within the images is not available and is too tedious to extract for large collection of images. To overcome this lack of labeled data, many recent developments use crowdsourcing services such as Amazon Mechanical Turk [19] to hire an on-demand human workforce over the internet to assign labels to data points. For instance, a tool named "Labelme" by MIT [20] could be used for this purpose. Similarly, Google started using its Captcha service, reCaptcha, to label address' digits collected from Street View images for the purpose of a deep neural network training [21]. An alternative and a relatively new framework of learning that tackles the inherent ambiguity better than supervised learning, is the Multiple Instance Learning (MIL) paradigm [22].

\subsection{Multiple Instance Learning}

Unlike standard supervised learning, in MIL, an object is not represented by a simple data point, but rather by a collection of instances, called a bag. Each bag can contain a different number of instances. In MIL, a bag is labeled negative if all of its instances are negative, and positive if at least one of its instances is positive (Note that positive bags may also contain negative instances). Positive bags can encode ambiguity since the instances themselves are not labeled. Given a training set of labeled bags, the goal of MIL is to learn a concept that predicts the labels of training data at the instance level and generalizes to predict the labels of testing bags and their instances [23].

The MIL problem was first formalized by Dietterich et al. [23] providing a solution to drug activity prediction. Ever since, it has increasingly been applied to a wide variety of tasks. Such as content-based information retrieval [24], drug discovery [25], pattern recognition [26], image classification [27], text classification [28], region-based image categorization [29], image annotation [30], object tracking [31] and time series prediction [22], to name a few. To illustrate the need for MIL, in the following we analyse how a multiple instance (MI) representation can be applied to image classification.

Consider the simple example of classifying images that contain "sky". In this problem, for an input image we want to determine whether a region that contains sky is present in the image. Using an MIL approach, each training image is represented by a bag of instances where each instance corresponds to a segmented region of interest. These regions could be obtained by dividing images into patches. A multiple instance representation is well suited for this purpose because only few regions may contain the object of interest (sky), that is the positive class. Other patches will be from background or other classes. This representation is illustrated in Figure 1 . Traditional, single instance learning are based on instance level (patch-level) labels and would require the image to be correctly segmented and labeled prior to learning.

\subsection{Mamdani Fuzzy Inference Systems}

A Mamdani Fuzzy Inference System (Mamdani-FIS) is a paradigm in soft computing which provides a means of approximate reasoning [32]. A Mamdani-FIS is capable of handling computing with knowledge uncertainty and measurements imprecision effectively [1]. It performs a non-linear mapping from an input space to an output space by deriving conclusions from a set of fuzzy if-then rules and known facts. Fuzzy rules are 


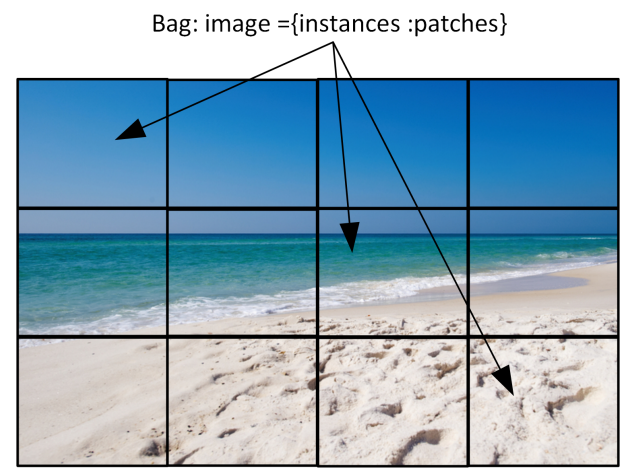

Figure 1. Example of an image represented as a bag of 12 instances. Each instance could be a feature vector extracted from one patch. The bag is labeled "sky" because at least one of its instances is sky. However, many other instances are not "sky". Labels at the instance level are not available.

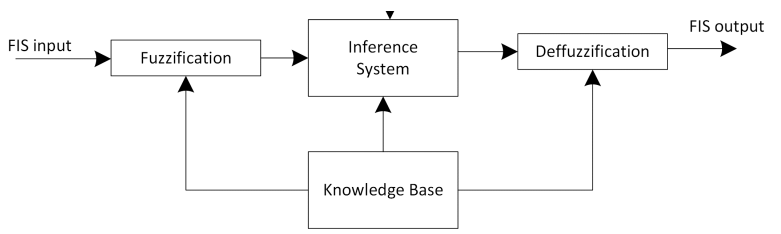

Figure 2. Main components of a typical Mamdani Fuzzy Inference System (Mamdani-FIS).

condition/action (if-then) rules composed of a set of linguistic variables (e.g. image patch). Each variable is assigned a linguistic term (e.g. red, green, blue). For example, the following rules could be used to identify patches from the image in Figure 1

- If patch is blue then region is sky.

- If patch is blue and patch position is upper half then region is sky.

- If patch is white and patch position is lower half then region is sand.

Typically, as shown in Figure 2, a Mamdani-FIS is composed of 5 components: (1) a Fuzzification unit that assigns a membership degree to each crisp input dimension in the input fuzzy sets; (2) a Knowledge Base characterized by fuzzy sets of linguistic terms; (3) a Rule Base containing a set of fuzzy if-then rules; (4) an Inference Unit that performs fuzzy reasoning; and (5) a Deffuzification Unit that generates crisp output values. Mamdani-FIS have proven to be very effective in various applications [3-5]. However, it is not applicable to cases where objects are represented by multiple instances.

\subsection{Motivations for Multiple Instance Mamdani Fuzzy In- ference}

There are two major limitations that prevent using standard Mamdani-FIS methods with multiple instance data. First, due to the absence of labels at the instance level, we cannot use standard Mamdani-FIS learning methods to construct the knowledge base. Second, we need an effective mechanism to aggregate instances' confidences and infer at the bag level.

The above limitations are due mainly to the inherent architecture of fuzzy inference systems. The generic Mamdani inference system shown in Figure 2 reasons with individual instances. First, the system's input is an individual instance. Second, the rules describe fuzzy regions within the instances space. Third, the output of the system corresponds to the fuzzy inference using a single instance. Fourth, labels of the individual instances are required when using learning techniques to identify the parameters of the system. In summary, traditional Mamdani-FIS cannot be used effectively within the MIL framework.

To address the above limitations. In this paper, we propose to generalize Mamdani fuzzy inference and extend it to reason with bags of instances. First, we develop multiple instance fuzzy logic that generalizes traditional fuzzy logic to compute with bags of instances. Under this work, we propose multiple instance generalization of fuzzy propositions, fuzzy if-then rules, fuzzy implication, and fuzzy reasoning. Next, we extend Mamdani-FIS to reason with bags instead of individual instances using the developed multiple instance fuzzy logic. We call the new inference system Multiple Instance-Mamdani (MI-Mamdani). Finally, we develop methods to identify and learn multiple instance fuzzy if-then rules from ambiguously labeled data.

\section{Related Work}

Zadeh introduced fuzzy sets in 1965 [33] and fuzzy logic in 1973 [3]. Mamdani and 36 followed with substantial additions [34-36]. Since then, many other developments and extensions to the fuzzy theory have been proposed. Most of the contributions can be classified under three categories: 1) contributions that propose variations and generalizations of fuzzy sets, 2) contributions that develop new fuzzy logic frameworks, and 3 ) contributions that propose new fuzzy inference schemes. For instance, Yager [37] introduced a new type of fuzzy sets known as fuzzy multisets (fuzzy bags), Atanassov [38] proposed intuitionistic fuzzy sets, and more recently Torra [39] proposed hesitant fuzzy sets. These approaches can be classified under 
the first category. Work that can be classified under the second category, include complex fuzzy logic [40] and complex fuzzy reasoning [41]. Under the third category, we can cite the contribution of Kaburlasos and Kehagias [42] that consisted of an extension of fuzzy inference systems based on lattice theory.

To the best of our knowledge, there have been no proposed variations that aimed at reformulating fuzzy logic to support reasoning with multiple instances at the same time. The only previous work, that has a mention of fuzzy and MIL in the same framework, was presented by Mahnot and Popescu [43]. They used fuzzy operators to compute diverse density [25]. This is by no means a reformulation of fuzzy logic to solve the multiple instance problem.

While there are no directly related approaches to our work, several methods are related as they aim to extend fuzzy logic and broaden its domain of applicability. For instance, fuzzy multisets [37] may seem to be related to our approach because it utilizes bags of elements to represent objects. A fuzzy multiset can be defined as a fuzzy set where multiple occurrences of an element are permitted. Within our framework it can be used to represent the results of bags' fuzzification; i.e., the membership degrees of each instance in a given fuzzy set. Also aggregations operators proposed for fuzzy multisets [44] could be used in our proposed extension. Other extensions of fuzzy sets could be adapted to the context of multiple instance fuzzy logic. For example, complex fuzzy sets [40] or complex fuzzy classes [45] are based on fuzzy sets characterized by complex-valued membership functions. Because of the two dimensionality nature of a complex fuzzy set, one can think of using it to carry reasoning with bags containing two instances at most. This later formulation is not necessary obvious and is worth investigating in future research projects.

\section{Multiple Instance Fuzzy Logic}

In this section, we formalize multiple instance fuzzy logic (MIFL). MIFL is different from traditional fuzzy logic in that it allows for an additional dimension of ambiguity and it enables fuzzy reasoning with bags of instances instead of a single instance at a time. We introduce multiple instance variations of fuzzy propositions, fuzzy if-then rules, and fuzzy reasoning, which are the building blocks of our proposed framework. The following formulation is inspired by the work of Jang et al. [47] on traditional fuzzy logic.

\subsection{Multiple Instance Fuzzy Propositions}

In traditional fuzzy logic, a fuzzy proposition can be written as

$$
p: X \text { is } A
$$

where $X$ receives values $x$ from a universal set $U$ and $A$ is a fuzzy set on $U$. An example of a proposition can be, "temperature is high". In traditional fuzzy logic, to evaluate the proposition $p$ in (1), $X$ is assigned a single value, say "temperature $=90$ ", this will lead to " $p$ : temperature $=$ 90 is high". This will work in most cases even if $X$ is a vector in $\mathbb{R}^{n}$. In fact, proposition (1) is valid as long as $X$ is expressed by a single instance. However, for MI data, the universe of discourse consists of bags of instances rather than single instances and the proposition needs to be generalized to a set of instances.

Let $B_{i}$ be a bag of $M_{i}$ instances. The $j$ th instance, $\mathbf{x}_{i j}$, is a $D$ dimensional vector with elements $x_{i j k}$ corresponding to features, i.e.,

$$
B_{i}=\left(\begin{array}{cccc}
x_{i 11} & x_{i 12} & \ldots & x_{i 1 D} \\
x_{i 21} & x_{i 22} & \ldots & x_{i 2 D} \\
\vdots & \vdots & \ddots & \vdots \\
x_{i M_{i} 1} & x_{i M_{i} 2} & \ldots & x_{i M_{i} D}
\end{array}\right) .
$$

Note that the number of instances can vary between bags ( $M_{i}$ depends on $B_{i}$ ). A bag is labeled positive if at least one of its instances is positive, and negative if all of its instances are negative. Let $\mathcal{B}=\left\{B_{i} \mid i=1, \ldots, N\right\}$ be the set of all bags. The universe of discourse $U$ is the set of all bags of a given problem $(U=\mathcal{B})$. For a given instance $\mathbf{x}_{i j}$ of a given bag $B_{i}$, we first define a "proposition instance" as

$$
p_{j}: \mathbf{x}_{i j} \text { is } A \text {, }
$$

Then, we define a multiple instance fuzzy proposition as the disjunction of proposition instances, i.e.,

$$
q: B_{i} \text { is } A \Longleftrightarrow q: \bigvee_{j=1}^{M_{i}} p_{j} \equiv \bigvee_{j=1}^{M_{i}}\left(\mathbf{x}_{i j} \text { is } A\right)
$$

In (4) " $V$ " is a T-conorm (maximum, algebraic sum, bounded sum, etc.), as defined in [47]. The proposition instance (" $\mathrm{x}_{i j}$ is $A$ ") is evaluated as in (11), and represents the degree of truth of the proposition on a single instance. Not only the bag has different forms of expression (or instances), the proposition itself has different instances of truth. It follows that the degree of truth 
of a multiple instance fuzzy proposition is a combination of degrees of truth associated with the proposition instances. (4) is analogues to fuzzy information fusion [49]. Fuzzy information fusion deals with merging uncertain observations that are possibly generated by heterogeneous sources. Thus, it is possible to view the combination of degrees of truth of multiple instances as a a fuzzy information fusion process. In the following, we formalize our truth instances fusion process.

Let $\tilde{\mu}_{A}\left(B_{i}\right)$ denote the degree of truth of a multiple instance fuzzy proposition. $\tilde{\mu}_{A}\left(B_{i}\right)$ indicates the "membership degree" of $B_{i}$ in $A$. The expression in (4) can be simplified further using the following theorem.

Theorem 3.1. Let $B$ be a collection of $M$ instances drawn form an instance space $X$, and let $A$ be a fuzzy set on $X$. The multiple instance proposition " $q: B$ is $A$ " is equivalent to the following

$$
q: B \text { is } A \Rightarrow \exists \mathbf{x} \in X \mid \tilde{\mu}_{A}(B)=\mu_{A}(\mathbf{x})
$$

Note that $\mathbf{x}$ is not necessary an instance of $B$.

From 4 , we have $\tilde{\mu}_{A}(B)=\bigvee_{j=1}^{M} \mu_{A}\left(\mathbf{x}_{j}\right)$, and we know that the T-conorm " $\bigvee$ ", aggregation operator, is closed in $[0,1]$. Thus, the aggregation of a given set of membership grades remains in $[0,1]$. It follows that $\tilde{\mu}_{A}(B)=\bigvee_{j=1}^{M} \mu_{A}\left(\mathbf{x}_{j}\right) \in[0,1]$. Assuming that the fuzzy set $A$ is normal and its membership function $\mu_{A}(x)$ is continuous, there exists $\mathbf{x} \in X$ such that

$$
\bigvee_{j=1}^{M} \mu_{A}\left(\mathbf{x}_{j}\right)=\mu_{A}(\mathbf{x})
$$

Hence,

$$
\bigvee_{j=1}^{M} \mu_{A}\left(\mathbf{x}_{j}\right)=\mu_{A}(\mathbf{x})=\tilde{\mu}_{A}(B)
$$

If the T-conorm is carried using a max operator, then $\tilde{\mu}_{A}\left(B_{i}\right)$ reduces to

$$
\tilde{\mu}_{A}\left(B_{i}\right)=\max \left\{\mu_{A}\left(\mathbf{x}_{i j}\right), j=1 \ldots M_{i}\right\}
$$

In $(8), \tilde{\mu}_{A}\left(B_{i}\right)$ is the highest degree of truth associated with the proposition's instances. This formulation is inline with the standard MIL assumption [22, 23], which states that a bag is positive if and only if one or more of its instances are positive. This relation will be covered in more details when we introduce multiple instance fuzzy inference in the next section.

\subsection{Multiple Instance Fuzzy If-Then Rules}

Recall that in traditional fuzzy logic a fuzzy if-then rule is expressed as

$$
\text { if } x \text { is } A \text { then } y \text { is } C
$$

where $A$ and $C$ are fuzzy sets on universes of discourse $X$ and $Y$, respectively. The rule in 9 combines the fuzzy propositions ( $x$ is $A$, $y$ is $C$ ) into a logical implication abbreviated as $A \rightarrow C$ with membership function $\mu_{A \rightarrow C}(x, y)$.

The rule is defined using a premise part that is a single instance traditional fuzzy proposition. To generalize to MI data, we define the multiple instance fuzzy rule as:

$$
\text { if } B_{i} \text { is A then } y \text { is } C \Longleftrightarrow \text { if } \bigvee_{j=1}^{M_{i}}\left(\mathbf{x}_{i j} \text { is } A\right) \text { then } y \text { is } C
$$

where $A$ and $C$ are fuzzy sets on the universes of discourse $X$ and $Y$, respectively. $B_{i}$ is a bag of instances $\mathbf{x}_{i j}$, and $M_{i}$ is the number of instances. The premise part of a multiple instance fuzzy rule (i.e., $\bigvee_{j=1}^{M_{i}}\left(\mathbf{x}_{i j}\right.$ is $\left.A\right)$ ) is a multiple instance proposition, whereas the consequence part is a traditional proposition. Since the premise part is a multiple instance proposition we will refer to this new logical implication as multiple instance implication. It is a fuzzy relation on the product space $\mathcal{B} \times C$ (B: bags' space). Formally,

$$
R=A \rightarrow C=A \times C=\int_{\mathcal{B} \times Y} \tilde{\mu}_{A}\left(B_{i}\right) \star \mu_{C}(y) /\left(B_{i}, y\right)
$$

where $\star$ is a T-norm and $A \times C$ is used to represent the fuzzy relation $R$

Lemma 3.2. There exists a transformation that transforms a multiple instance fuzzy implication to a traditional fuzzy implication.

Using Theorem (3.1) we replace $\tilde{\mu}_{A}\left(B_{i}\right)$ by $\mu_{A}(x)$ and rewrite (11) as

$$
R=A \rightarrow C=A \times C=\int_{X \times Y} \mu_{A}(x) \star \mu_{C}(y) /(x, y)
$$

which is the expression of a traditional fuzzy relation. Thus, multiple instance fuzzy implication can be carried using traditional fuzzy implication.

In (11), $R$ has a membership function, denoted $\mu_{A \rightarrow C}\left(B_{i}, y\right)$, that represents the degree of truth of the implication when $\mathcal{B}$ is equal to $B_{i}$ and $Y$ is equal to $y$. For instance, using $\min$ and 
product as implication operators, we have:

$$
\begin{aligned}
\mu_{A \rightarrow C}\left(B_{i}, y\right)=\int_{\mathcal{B} \times Y} \tilde{\mu}_{A}\left(B_{i}\right) & \wedge \mu_{B}(y) /(x, y)= \\
& \min \left[\tilde{\mu}_{A}\left(B_{i}\right), \mu_{C}(y)\right]
\end{aligned}
$$

and,

$$
\begin{aligned}
& \mu_{A \rightarrow C}\left(B_{i}, y\right)= \\
& \quad \int_{\mathcal{B} \times Y} \tilde{\mu}_{A}\left(B_{i}\right) \cdot \mu_{C}(y) /(x, y)=\tilde{\mu}_{A}\left(B_{i}\right) \cdot \mu_{C}(y)
\end{aligned}
$$

\subsection{Multiple Instance Fuzzy Reasoning}

Multiple instance fuzzy reasoning is needed when the universe of discourse $U$ is a "bag-space" $(U=\mathcal{B})$, i.e., every input element is a bag of instances rather than a single instance. In this case, we define the Generalized Modus Ponens as

$$
\begin{aligned}
& \text { premise: if } B_{i} \text { is } A \text { then } y \text { is } C \Longleftrightarrow \text { if } \bigvee_{j=1}^{M_{i}}\left(X_{i j} \text { is } A\right) \text { then } y \text { is } C \\
& \text { fact: } B_{i}=\left\{X_{i j}\right\}_{j=1}^{M_{i}} \text { and } X_{i 1} \text { is } A_{1}^{\prime}, X_{i 2} \text { is } A_{2}^{\prime}, \ldots, X_{i M} \text { is } A_{M_{i}}^{\prime} \\
& \hline \text { consequence: } y \text { is } C^{\prime}
\end{aligned}
$$

$A$ and $\left\{A_{j}^{\prime}\right\}_{i=j}^{M_{i}}$ are fuzzy sets on $X$ (the instances space), and $C$ is a fuzzy set on $Y$. Using the composition rule of inference [3], we determine $C^{\prime}$ using

$$
C^{\prime}=\left(\bigvee_{j=1}^{M_{i}} A_{j}^{\prime}\right) \circ(A \rightarrow C)=\bigvee_{j=1}^{M_{i}}\left(A_{j}^{\prime} \circ(A \rightarrow C)\right)
$$

and we have,

$$
\mu_{C^{\prime}}(y)=\bigvee_{j=1}^{M_{i}}\left\{\max _{x}\left(\min \left[\mu_{A_{j}^{\prime}}(x), \mu_{A \rightarrow C}(x, y)\right]\right)\right\}
$$

Using min as implication operator, 16 is equivalent to

$$
\mu_{C^{\prime}}(y)=\bigvee_{j=1}^{M_{i}}\left\{\max _{x}\left(\min \left[\mu_{A_{j}^{\prime}}(x), \min \left(\mu_{A}(x), \mu_{C}(y)\right)\right]\right)\right\}
$$

further simplification yields

$$
\mu_{C^{\prime}}(y)=\min \left[\bigvee_{j=1}^{M_{i}}\left\{\max _{x}\left(\min \left[\mu_{A_{j}^{\prime}}(x), \mu_{A}(x)\right]\right)\right\}, \mu_{C}(y)\right]
$$

For instance, if the "max" aggregation operator is used, we have

$$
\mu_{C^{\prime}}(y)=\min \left[\max \left\{\max _{x}\left(\min \left[\mu_{A_{j}^{\prime}}(x), \mu_{A}(x)\right]\right)\right\}_{j=1}^{M_{i}}, \mu_{C}(y)\right]
$$

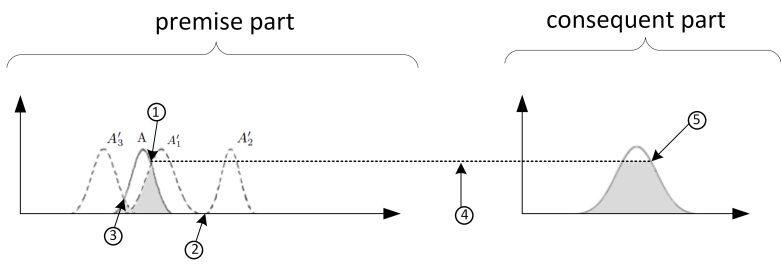

Figure 3. Illustration of the multiple instance inference process using the "max" aggregation operator. Legend: $(1)=\max _{x}\left(\min \left(\mu_{A_{1}^{\prime}}(x)\right.\right.$, $\left.\left.\mu_{A}(x)\right)\right), \quad(2)=\max _{x}\left(\min \left(\mu_{A_{2}^{\prime}}(x), \mu_{A}(x)\right)\right), \quad(3)=$ $\max _{x}\left(\min \left(\mu_{A_{3}^{\prime}}(x), \mu_{A}(x)\right)\right),(4)=\max _{2} \max _{x}\left(\min \left[\mu_{A_{j}^{\prime}}(x)\right.\right.$, $\left.\left.\left.\mu_{A}(x)\right]\right)\right\}_{j=1}^{3},(5)=\mu_{C^{\prime}}(y)$

The term " $\max \left\{\max _{x}\left(\min \left[\mu_{A_{j}^{\prime}}(x), \mu_{A}(x)\right]\right)\right\}_{j=1}^{M_{i}}$ ” in 19 can be interpreted as the rule firing strength [46].

To summarize, the proposed multiple instance fuzzy reasoning involves the following 3 main steps:

1. Compute the multiple instance proposition degree of truth, i.e., evaluate $\max \left\{\mu_{A^{\prime}}\left(\mathbf{x}_{i j}\right), j=1 \ldots M_{i}\right\} ;$

2. Compute the rule firing strength, or the degree of belief for the antecedent part;

3. Compute the degree of belief of the consequent part by applying the "min" operator.

Multiple instance fuzzy logic is a generalization of fuzzy logic, in fact if we set the number of instances in each bag to 1 , all presented approaches will reduce to those of traditional fuzzy logic.

\subsection{Illustrative Example}

Let $B$ be a bag of three instances $\mathbf{x}_{1}, \mathbf{x}_{2}$, and $\mathbf{x}_{3}$. Let $A_{1}^{\prime}, A_{2}^{\prime}$, $A_{3}^{\prime}$ be the fuzzy sets associated with the instances. Given this fact we want to evaluate the following multiple instance rule

$$
\text { if } B \text { is A then } y \text { is } C \Longleftrightarrow \text { if } \bigvee_{j=1}^{3}\left(\mathbf{x}_{j} \text { is } A\right) \text { then } y \text { is } C
$$

where A and C are fuzzy sets. Figure 3 illustrates the proposed multiple instance inference process. To compute the rule firing strength we need to evaluate

$$
\mu_{C^{\prime}}(y)=\min \left[\max \left\{\max _{x}\left(\min \left[\mu_{A_{j}^{\prime}}(x), \mu_{A}(x)\right]\right)\right\}_{j=1}^{3}, \mu_{C}(y)\right]
$$

First, we compute the truth instances (the shaded area in the premise part of Figure 3). Then, all truth instances are aggregated using the "max" operator, i.e., we select the highest truth 
instance as the rule firing strength. Finally, the membership function (MF), $\mu_{C^{\prime}}(y)$, for the consequent part is computed as the MF of $C$ clipped by the rule firing strength.

\subsection{Discussion}

Equation (18) defines fuzzy reasoning with bags of instances. To reach this goal, we have proposed multiple instance variations of fuzzy logic building blocks; i.e., propositions, if-then rules, implications, and Generalized Modus Ponens. Our generalization was derived using a thoroughly and abstract mathematical formulation. The new findings will be used to build more advanced and complex fuzzy inference systems as will be shown in the next section.

The difference between our multiple instance framework and fuzzy logic may seem subtle, but there is an important contribution to point out. In his short abstract published in 2008, titled "Is there a need for fuzzy logic?" [49], Zadeh wrote: "Fuzzy logic is not fuzzy. Basically, fuzzy logic is a precise logic of imprecision and approximate reasoning". We think that fuzzy logic is powerful at modeling knowledge uncertainty and measurements imprecision. More generally, it is one of the best frameworks to model vagueness. However, in addition to uncertainty and imprecision, there is a third vagueness concept that fuzzy logic does not address quiet well, yet. This vagueness concept is due to the ambiguity that arises when the data have multiple forms of expression, this is the case for multiple instance problems. Our framework deals with ambiguity by introducing the novel concept of truth instances: when carrying reasoning using MIFL, a proposition will not only have one degree of truth, it will have multiple degrees of truth, we call truth instances. Thus, effectively encoding the third vagueness component of ambiguity and increasing the expressive power of traditional fuzzy logic.

\section{Multiple Instance Mamdani Fuzzy Inference}

The traditional Mamdani inference system [35] is limited to reason with individual instances and cannot be used effectively within the context of multiple instance learning.

In the following, we propose a generalization of Mamdani fuzzy inference to extend it to reason with bags of instances. Similar to the traditional Mamdani system, we formulate the proposed MI-Mamdani by means of multiple instance fuzzy if-then rules that can evaluate bags. As introduced in Section 3.2. multiple instance fuzzy rules can be expressed using (22).
In multiple instance fuzzy reasoning, the antecedent part, $\bigvee_{j=1}^{M_{p}}$ (If $x_{p j 1}$ is $A_{1}^{i} \ldots$, and $x_{p j D}$ is $A_{D}^{i}$ ), evaluates the degree to which the antecedent fuzzy sets describe each instance separately, then all responses are combined into a rule firing strength using a T-conorm. Using this inference style, the rule will be fired if and only if there exist at least one instance in the bag that can be described by means of the antecedent fuzzy sets.

The reason behind using a T-conorm for combining individual instances' responses goes back to the standard MIL assumption [22, 23] which states that each instance has a hidden class label, and under this assumption, an example is positive if and only if one or more of its instances are positive. Thus, the bag-level class label is determined by the disjunction of the instance-level class labels. In the context of multiple instance inference, if a fuzzy rule describes a local region of the instances space that happens to be a positive MIL concept, and if the rule's output is high, the multiple instance fuzzy rule will be capable of classifying positive bags correctly. This is because at least one instance from each positive bag will activate the rule, leading to a high output (positive label). On the other hand, negative bags will not be able to significantly activate any rule. Figure 4 illustrates the proposed MI-Mamdani system and its fuzzy inference mechanism to derive the output $\mathrm{z}$ in response to a bag of instances for the simple case of two rules.

As it can be seen, the premise part of the rules evaluates all the bag's instances simultaneously. The inference starts by the fuzzification of instances $x_{p m}$ of a given bag $B_{p}$. Fuzzification assigns a membership degree to each input instance dimension in the rules input fuzzy sets. In Figure 4 instance $x_{p m}$ activates the $i$ th input fuzzy set of the $j$ th rule by a degree of truth $w_{m i j}$. Next, an implication process is executed to combine the activations of the instances within the bag resulting in the activation of the rules' output with different degrees. In this example, we use a simple min operator, and the output of rule $R^{j}$ will be partially activated by a degree $w_{m j}=\min _{k=1, \ldots, D} w_{m k j}$. The $w_{m j}$ (truth instances) are combined in the premise part using the max T-conorm, resulting in the activation of rule $R^{j}$ by a degree $w_{j}=\max _{m=1, \ldots, M} w_{m j}$. Next, using a simple max operator, the 2 output fuzzy sets are aggregated to generate one output fuzzy set. Finally, the output set is defuzziffied (e.g., using its centroid) to generate a final crisp output value.

The MI-Mamdani inference system allows the use of different T-conorms on different rules. The choice of the appropriate function should depend on the application and the purpose of the rule. More specifically: should the rule be activated if at least one instance of the bag is within the target concept? 
$\mathcal{R}^{i}\left(\mathbf{B}_{p}\right): \bigvee_{j=1}^{M_{p}}\left(\right.$ If $x_{p j 1}$ is $A_{1}^{i}$ and $x_{p j 2}$ is $A_{2}^{i}, \ldots$, and $x_{p j D}$ is $\left.A_{D}^{i}\right)$, then $o^{i}$ is $C^{i}$

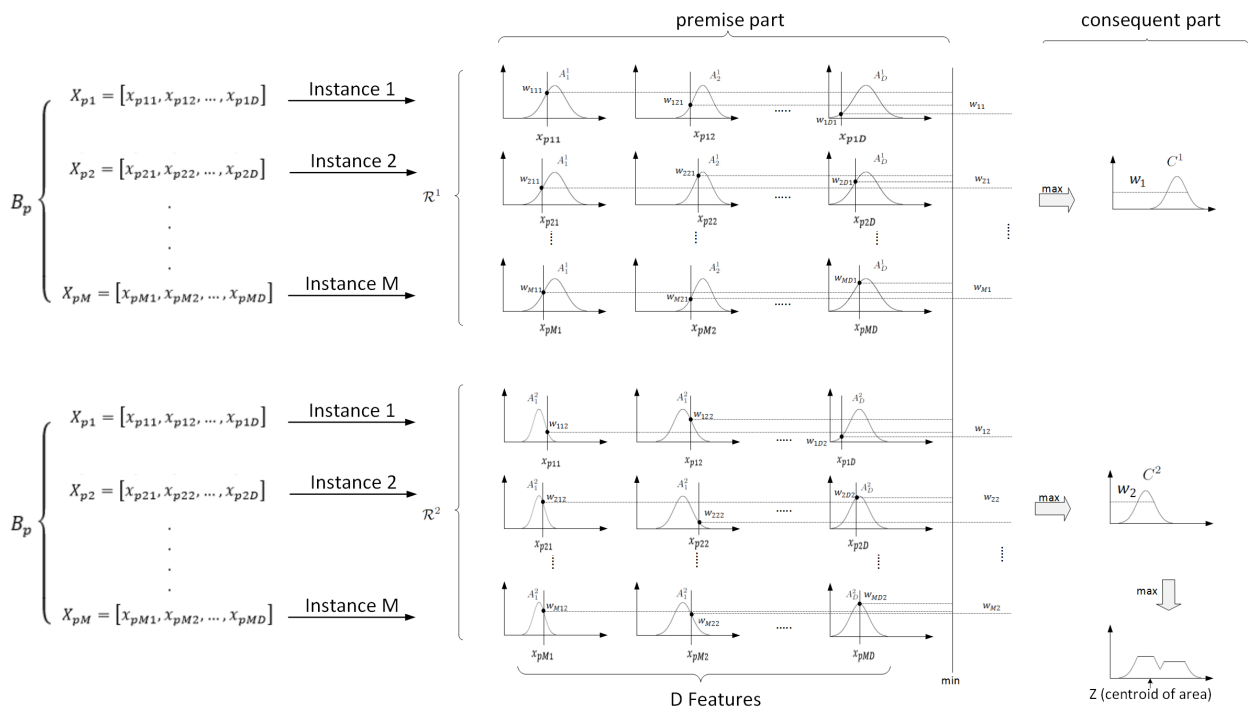

Figure 4. Illustration of the proposed Multiple Instance Mamdani Fuzzy Inference System.

Or should it be activated only if at least a fixed subset of the instances are within the target concept?

Finally, we should note here that if we set $M_{p}$ to 1 (i.e., constraint all bags to include only one instance), (22) reduces to a traditional fuzzy if-then rule commonly used in Mamdani FIS. Thus, the proposed MI-Mamdani fuzzy inference system, can be considered a generalization of the traditional Mamdani system.

\subsection{Learning the Structure and Parameters of MI-Mam- dani}

The most important task in fuzzy inference with MI-Mamdani is the identification and learning of the system's structure and its parameters. Structure identification consists of identifying the number of multiple instance if-then fuzzy rules, identifying the MFs of the premise and consequent parts, and also the T-conorms involved in the multiple instance fuzzy reasoning. After structure, the parameters of the MFs need to be learned.

The system's structure and parameters identification rely mainly on determining the characteristics of the local regions within the instances' space that characterize positive bags. In traditional (i.e., single instance representation) fuzzy modeling, this task is achieved through input space partitioning, typically using grid partitioning and clustering [46]. In multiple instance inference systems, we need to identify regions that are defined by positive instances, referred to as positive concepts. Since in MIL, data is labeled at the bag level and not at the instance level, traditional space partitioning methods could not be used to learn the multiple instance fuzzy rules.

In the following, we describe our proposed approach to identify multiple instance fuzzy rules based on a fuzzy clustering algorithm of multiple instance (FCMI) data [50]. FCMI identifies target concepts that correspond to dense regions in the instance space that include as many positive instances as possible and as few negative instances as possible. In particular, we define the permise parts of the MI-FIS rules as local contexts within the input space (instances' space) that coincide with the identified target concepts.

Assume that we have $\mathrm{N}$ training bags, $\mathcal{B}=\left\{B_{i} \mid i=1, \ldots, N\right\}$, and the set of their corresponding labels, $\mathcal{T}=\left\{t_{i} \mid i=1, \ldots, N\right\}$. Let $\mathbf{T}=\left\{C_{1}, \ldots, C_{r}\right\}$, be $r$ target concept points. Each target concept $C_{i}$ is characterized by a center $c_{i} \in \mathbb{R}^{D}$ and a feature relevance scale vector $s_{i} \in \mathbb{R}^{D}$. The FCMI algorithm maximizes a fuzzy Multiple Concept Diverse Density (MDD) measure [50] defined as:

$$
M D D(\mathbf{T}, \mathbf{U})=\prod_{n=1}^{N} \prod_{i=1}^{r}\left[\operatorname{Pr}\left(C_{i} \mid B_{n}\right)\right]^{u_{i n}^{m}}
$$


In 28, $\mathbf{U}=\left[u_{i n}\right]$ is a membership matrix such that each bag $B_{n}$ is assigned to target concept $C_{i}$ with membership degree $u_{i n}$, and $m$ is a fuzzifier that controls the fuzziness of the partitions as in the FCM [51]. $\operatorname{Pr}\left(C_{i} \mid B_{n}\right)$ is the probability that $C_{i}$ is a target concept given $B_{n}$, and defined as

$$
\operatorname{Pr}\left(C_{i} \mid B_{n}\right)=\left\{\begin{array}{c}
1-\prod_{k=1}^{M}\left(1-\operatorname{Pr}\left(x_{n k} \in C_{i}\right)\right) \text { if } l\left(B_{n}\right)=1 \\
\prod_{k=1}^{M}\left(1-\operatorname{Pr}\left(x_{n k} \in C_{i}\right)\right) \text { if } l\left(B_{n}\right)=0
\end{array}\right.
$$

where $l\left(B_{n}\right)$ is the label of bag and $x_{n k}$ is the $k$ th instance of bag $B_{n} . \operatorname{Pr}\left(X_{n k} \in C_{i}\right)$ is regarded as the similarity of instance $X_{n k}$ to target concept $C_{i}$, and its computed using

$$
\operatorname{Pr}\left(X_{n k} \in C_{i}\right)=e^{-\left(\sum_{j=1}^{D} s_{i j}\left(x_{n k j}-c_{i j}\right)^{2}\right)}
$$

In (25), $s_{i j}$ is a scaling parameter that weights the role of feature $j$ in target concept $i[25]$.

Let $\left\{C_{i}^{o p t}=\left\{c_{i}^{o p t}, s_{i}^{\text {opt }}\right\}\right\}_{i=1}^{r}$ be the optimal target concepts identified by FCMI that maximizes 28). Let $\mathbf{T}=\left\{C_{1}, \ldots, C_{r}\right\}$, be the $r$ target concept points. For simplicity, we assume that the MFs of the $r$ multiple instance rules are Gaussian MFs, with centers $c_{i j}, i=1, \ldots, r$, and $j=1, \ldots, D$. For a given multiple instance rule $\mathcal{R}^{i}$, the centers of the premise part's MFs are the centers of the target concepts, i.e.,

$$
c_{i j}=C_{i j}, \text { for } j=1, \ldots, D
$$

The diverse density of each concept decreases gradually as we move away from $C_{i}$. Intuitively, the width $\sigma_{i j}$ of a given concept $C_{i}$ along dimension $j$ can be set to the radius beyond which $M D D$ is lower than a diverse density threshold $\tau_{i}$. Formally, the standard deviations, $\left\{\sigma_{i j}\right\}$, can be computed as following:

$$
\sigma_{i j}=\min _{Z \in \mathcal{I}}\left\{\left|C_{i j}-Z_{j}\right| \text { s.t. } M D D_{i}(Z)<\tau_{i}\right\},
$$

In (27), $\mathcal{I}$ is the set of all instances, $Z$ is a $D$ dimensional vector and $\tau_{i}$ is constant, typically

$$
\tau_{i}=\frac{1}{2} M D D_{i}\left(C_{i}\right)=\prod_{n=1}^{N}\left[\operatorname{Pr}\left(C_{i} \mid B_{n}\right)\right]^{u_{i n}^{m}} .
$$

To identify the rules' consequent parts we can employ one of the following two strategies:

1. The consequents parts of multiple instance fuzzy rules are set to the singleton fuzzy set $\{1\}$. Using this strategy, positive bags that activate a rule, lead to rule's output of 1. This is inline with standard MIL assumption given that rules describe positive concepts.
2. Treat concepts as regular contexts. For each multiple instance fuzzy rule, its consequents fuzzy sets' parameters are identified by considering the ratio of positive to negative instances within the context described by the multiple instance fuzzy rule. For example, if a context has $90 \%$ instances from positive bags, then a consequent MF can be set to a Gaussian with center equals to 0.9 and a predefined standard deviation $\varepsilon$.

\section{Experimental Results}

\subsection{Synthetic Data}

To illustrate the proposed multiple instance fuzzy rules and the ability to learn from data without instance-level labels, we use a simple synthetic dataset. The data were generated from a distribution of two positive contexts, marked with squares in Figure 5 From each positive concept we generated 50 bags. We also generated 50 negative bags randomly from non-concept regions. The number of instances within each bag is a random number between 2 and 10 instances. The data is shown in Figure 5. Instances from negative bags are shown as ".", and instances from positive bags are shown as " + " or " $\Delta$ " depending on the underlying concept. In Figure 5, we highlight one bag from Concept 1 by circling all of its instances. As it can be seen, one instance is close to a dense region of a positive concept while the other instances are scattered around. We note here that the centers of concepts in Figure 5 are shown just for the purpose of explanation and validation. We do not use this information as it is not available.

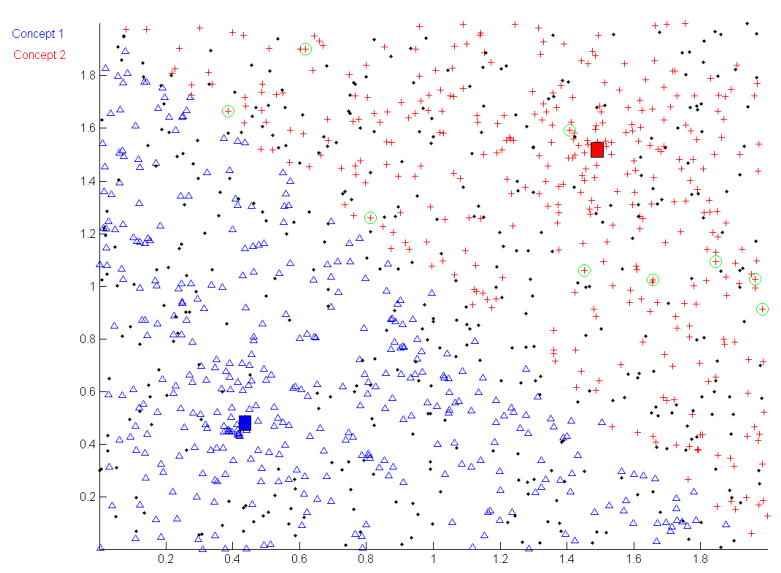

Figure 5. Instances from positive and negative bags drawn from data that have 2 concepts. Instances from negative bags are shown as ".", and instances from positive bags are shown as "+" or " $\triangle$ ". Instances from one sample positive bag are circled. 


\subsubsection{Rules learning}

As highlighted in Section 4.1. first, we run FCMI [50] to identify target concepts. These points are then used to identify the parameters of the fuzzy rules.

Next, for the rules' consequents identification, we set the output MFs to the singleton fuzzy set $\{1\}$. This will ensure that bags that have instances within the positive concepts will get assigned high output. Finally, all the rules' parameters are used within an MI-Mamdani fuzzy inference system composed of two multiple instance fuzzy rules, each with two inputs and one output. A graphical representation of this system is shown in Figure 6 It can be seen that the centers of MFs identified using FCMI match the centers of positive concepts shown in Figure 5 To test the system, we generate 3 bags of instances: 2 positive bags and 1 negative bag. The instances of these bags are displayed in Figure 7 The multiple instance fuzzy inference using the MI-Mamdani system of the 3 test bags is summarized in Figure 8 The inference starts by fuzzification of all the instances at the same time, as illustrated in Figure 8(a) then multiple instance fuzzy implication process is executed resulting in the activation of the rules' output with different degrees (each degree of activation is a firing strength as defined in Section 3.3. Next, using a simple max operator, the 2 output fuzzy sets are aggregated to generate one output fuzzy set. Finally, the output set is defuzzified using its centroid weighted by the maximum rule firing strength. The weighting ensures that negative bags that do not activate any rule and will always have a low output. In addition, we notice that while both first and second bags are positive, the inference process assigned a lower degree of belief to the second bag and as a consequence a lower output value. This will not impact classification's results as negative bags will not be able to activate any of the rules with a significant degree. But it will rather give applications an
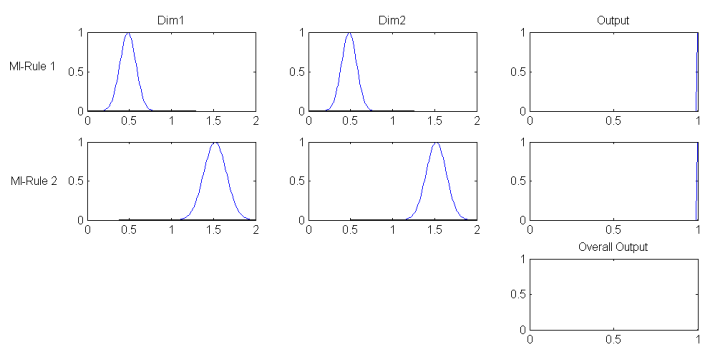

Figure 6. Illustration of Multiple Instance Mamdani Fuzzy Inference System learned using fuzzy clustering algorithm of multiple instance (FCMI).

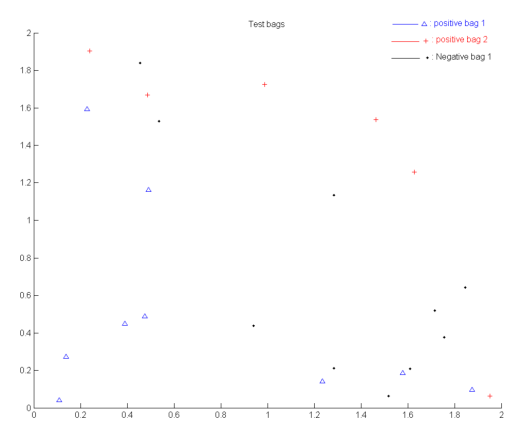

Figure 7. Instances from 2 positive and 1 negative bag.

assessment about the confidence of the prediction.

\subsection{Fusion of Multiple Landmine Detection Algorithms Using MI-Mamdani}

In the following, we apply the proposed multiple instance inference to fuse the output of multiple discrimination algorithms for the purpose of landmine detection using Ground Penetrating Radar (GPR). GPR data collected at different locations and different dates were used to train and test the proposed fusion method. The alarm collection covers a variety of anti-tank mines including 319 encounters of anti-tank with high metal content (ATHM) and 422 encounters of anti-tank with low metal content (ATLM). The fusion training data are grouped into bags. Each bag represents a GPR alarm and has 15 instances extracted at multiple depths. Each instance include 4 features: two discrimination algorithms and two auxiliary features. The discrimination algorithms are based on the standard Edge Histogram Descriptor (EHD) algorithm proposed by Frigui and Gader [52]. The first one, called EHDDT, extracts information of the signal from the down-track direction. The second discrimination algorithm, called EHDCT, extracts information for the cross-track direction. The auxiliary features are synthesized from the radar signal at certain depths. In particular, SignatureWidth for down-track and SignatureWidth for cross-track are used to capture the effective width of the strong components within the GPR signal along (depth, down-track) slices and the width along (depth, cross-track) slices. Labels for the bags are available as binary ground truth: target/nontarget (positive/negative). This formulation fits perfectly the MIL paradigm.

To learn an MI-Mamdani system from the training data (bags) for the purpose of fusion of discrimination algorithms, first, we apply FCMI to extract concept points. Next, we generate 


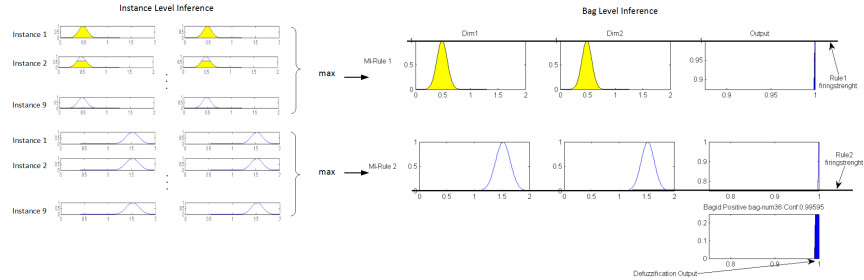

(a)

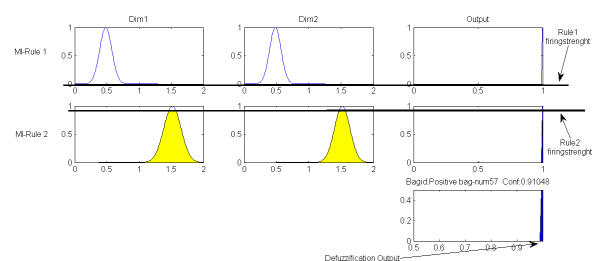

(b) .

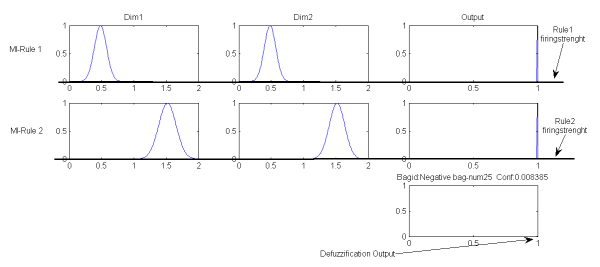

(c)

Figure 8. Multiple instance fuzzy inference using the learned Multiple Instance Mamdani (MI-Mamdani) system. The level of the activation indicates the membership degree of a bag in a given concept (i.e., rule). The system defuzzified output is the final confidence value. (a) Inference process with the first positive bag. (b) Inference process with the second positive bag. (c) Inference process with the negative bag.

multiple instance fuzzy rules from concept points as outlined in Section 4.1. Finally, the learned rules are combined into an MI-Mamdani multiple instance fuzzy inference system.

The individual discriminators and the proposed fusion were trained and tested using 10-folds cross validation. Figure 9 displays the ROC's of all methods. To provide a qualitative evaluation of the proposed fusion method, we compare its performance to a fusion method based on the standard Mamdani [13]. Since the standard Mamdani cannot learn from partially labeled data, an expert is used to label the instances. We also compare MI-Mamdani performance to a global fusion method based on geometric mean (GeoMean) and a naive MIL implementation of Mamdani (NaiveMamdani) where all instances from positive bags are considered positive and all instances from negative bags are considered negative.

Overall, MI-Mamdani outperformed the individual discriminators (EHDDT and EHDCT), the NaiveMamdani and Ge-

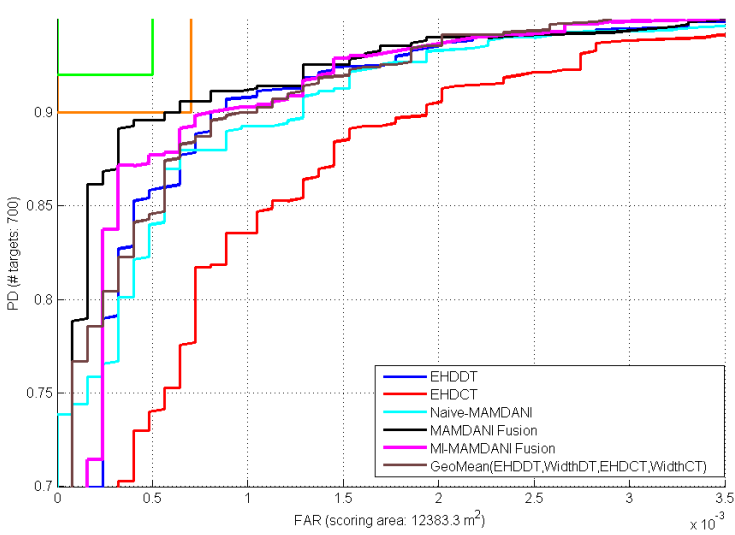

Figure 9. Comparison of the individual discriminators, the proposed Multiple Instance Mamdani (MI-Mamdani), Mamdani, NaiveMamdani and GeoMean fusion methods.

oMean fusion methods. This is due to the ability of MI-Mamdani to overcome labeling ambiguity by generating a fuzzy representation of concepts. The standard Mamadani fusion method performed the best - which is expected - given the additional information available at the instances' level to this later fusion method.

\section{Conclusions}

In this paper, we have introduced a new framework to accomplish fuzzy inference with multiple instance data. Our work generalizes traditional fuzzy logic to enable reasoning with bags rather than single instances. In particular, we have introduced multiple instance variations of fuzzy propositions, fuzzy implication, fuzzy if-then rules, and fuzzy reasoning. Our generalization was derived using a thoroughly and abstract mathematical formulation. We also used our multiple instance fuzzy logic framework to derive a MI-Mamdani fuzzy inference system. We have also presented a method to learn multiple instance rules from data to solve MIL problems. The FCMI algorithm is used to extract concept points in the instances' space which are then transformed into multiple instance rules. This approach is essentially based on intuition. Although premise and consequent parameters of the MI-Mamdani system can be learned from data, the processes of identifying both set of parameters are independent.

In future work, we will introduce a neuro-fuzzy architecture capable of learning from ambiguously labeled data without having to use FCMI to identify multiple instance rules, and 
can jointly learn the set of the optimal premise and consequent parameters using alternating optimization.

\section{Conflict of Interest}

No potential conflict of interest relevant to this article was reported.

\section{References}

[1] L. A. Zadeh, A Theory of Approximate Reasoning (AR). Berkeley, CA: Electronics Research Laboratory, College of Engineering, University of California, 1977.

[2] O. Cordon, "A historical review of evolutionary learning methods for Mamdani-type fuzzy rule-based systems: designing interpretable genetic fuzzy systems," International Journal of Approximate Reasoning, vol. 52, no. 6, pp. 894913, 2011. http://dx.doi.org/10.1016/j.ijar.2011.03.004

[3] L. A. Zadeh, "Outline of a new approach to the analysis of complex systems and decision processes," IEEE Transactions on Systems, Man and Cybernetics, vol. 3, no. 1, pp. 28-44, 1973. http://dx.doi.org/10.1109/tsmc.1973. 5408575

[4] E. H. Mamdani, "Application of fuzzy algorithms for control of simple dynamic plant," Proceedings of the Institution of Electrical Engineers, vol. 121, no. 12, pp. 15851588, 1974. http://dx.doi.org/10.1049/piee.1974.0328

[5] R. Jager, H. Verbruggen, and P. M. Bruijin, "Fuzzy inference in rule-based control systems," in Proceedings of 1st International Conference on Intelligent Systems Engineering, Edinburgh, Scotland, 1992, pp. 232-237.

[6] C. C. Lee, "Fuzzy logic in control systems: fuzzy logic controller. II," IEEE Transactions on Systems, Man and Cybernetics, vol. 20, no. 2, pp. 419-435, 1990. http://dx. doi.org/10.1109/21.52552

[7] J. Casillas, Interpretability Issues in Fuzzy Modeling. Berlin: Springer-Verlag, 2003.

[8] C. Y. Chiu, H. C. Lin, and S. N. Yang, "A fuzzy logic CBIR system," in Proceedings of the 12th IEEE International Conference on Fuzzy Systems, St. Louis, MO, 2003, pp. 1171-1176. http://dx.doi.org/10.1109/fuzz.2003. 1206597
[9] A. A. Othman, H. R. Tizhoosh, and F. Khalvati, "EFIS: evolving fuzzy image segmentation," IEEE Transactions on Fuzzy Systems, vol. 22, no. 1, pp. 72-82, 2014. http: //dx.doi.org/10.1109/tfuzz.2013.2246761

[10] D. Y. Kim and H. M. Chung, "Image recognition by learning multi-valued logic neural network," International Journal of Fuzzy Logic and Intelligent Systems, vol. 2, no. 3, pp. 215-220, 2002. http://dx.doi.org/10.5391/ijfis.2002.2. 3.215

[11] H. K. Kwan and L. Y. Cai, "Supervised fuzzy inference network for invariant pattern recognition," in Proceedings of the 43rd IEEE Midwest Symposium on Circuits and Systems, Lansing, MI, 2000, pp. 850-854. http://dx.doi. org/10.1109/mwscas.2000.952888

[12] J. S. Kim, "Prediction of user's preference by using fuzzy rule \& RDB inference: a cosmetic brand selection," International Journal of Fuzzy Logic and Intelligent Systems, vol. 5, no. 4, pp. 353-359, 2005. http: //dx.doi.org/10.5391/ijfis.2005.5.4.353

[13] A. Khalifa and H. Frigui, "Fusion of multiple algorithms for detecting buried objects using fuzzy inference," Proceedings of SPIE, vol. 9072, 2014. http://dx.doi.org/10. $1117 / 12.2051217$

[14] M. Y. Chen and D. Linkens, "Rule-base self-generation and simplification for data-driven fuzzy models," in Proceedings of the 10th IEEE International Conference on Fuzzy Systems, Melbourne, Australia, 2001, pp. 424-427. http://dx.doi.org/10.1109/fuzz.2001.1007339

[15] D. Z. Saletic, "On data-driven procedure for determining the number of rules in a Takagi-36 fuzzy model," in Proceedings of the International Conference on Computer as a Tool (EUROCON2005), Belgrade, Serbia 2005, pp. 1132-1135. http://dx.doi.org/10.1109/eurcon. 2005.1630151

[16] P. Zikopoulos, D. DeRoos, K. Parasuraman, T. Deutsch, J. Giles, and D. Corrigan, Harness the Power of Big Data: The IBM Big Data Platform. New York, NY: McGrawHill, 2012.

[17] “Flickr,” 2014; Available http://www.flickr.com/

[18] “Craigslist," 2014; Available http://www.craigslist.org 
[19] A. Sorokin and D. Forsyth, "Utility data annotation with Amazon Mechanical Turk," in Proceedings of IEEE Computer Society Conference on Computer Vision and Pattern Recognition Workshops, Anchorage, AK, 2008, pp. 1-8. http://dx.doi.org/10.1109/cvprw.2008.4562953

[20] A. Torralba, B. C. Russell, and J. Yuen, "Labelme: online image annotation and applications," Proceedings of the IEEE, vol. 98, no. 8, pp. 1467-1484, 2010. http://dx.doi. org/10.1109/jproc.2010.2050290

[21] I. J. Goodfellow, Y. Bulatov, J. Ibarz, S. Arnoud, and V. Shet, "Multi-digit number recognition from street view imagery using deep convolutional neural networks," in Proceedings of 2nd International Conference on Learning Representations (ICLR2014), Banff, Canada, 2014, pp. 1-13.

[22] O. Maron, "Learning from ambiguity," Ph.D. dissertation, Massachusetts Institute of Technology, Cambridge, MA, 1998.

[23] T. G. Dietterich, R. H. Lathrop, and T. Lozano-Perez, "Solving the multiple instance problem with axis-parallel rectangles," Artificial Intelligence, vol. 89, no. 1-2, pp. 31-71, 1997. http://dx.doi.org/10.1016/s0004-3702(96) 00034-3

[24] C. Zhang, X. Chen, and W. B. Chen, "An online multiple instance learning system for semantic image retrieval," in Proceedings of 9th IEEE International Symposium on Multimedia Workshops (ISMW'07), Taichung, Taiwan, 2007, pp. 83-84. http://dx.doi.org/10.1109/ism.workshops. 2007.23

[25] O. Maron and T. Lozano-Perez, "A framework for multiple-instance learning," in Proceedings of the 1997 Conference on Advances in Neural Information Processing Systems (NIPS'97), Cambridge, MA, 1998, pp. 570576.

[26] A. Karem and H. Frigui, "A multiple instance learning approach for landmine detection using ground penetrating radar," in Proceedings of 2011 IEEE International Geoscience and Remote Sensing Symposium (IGARSS), Vancouver, Canada, 2011, pp. 878-881. http://dx.doi.org/ 10.1109/igarss.2011.6049271

[27] R. Rahmani and S. A. Goldman, "MISSL: Multipleinstance semi-supervised learning," in Proceedings of the 23rd International Conference on Machine Learning (ICML'06), Pittsburgh, PA, 2006, pp. 705-712. http: //dx.doi.org/10.1145/1143844.1143933

[28] S. Ray and M. Craven, "Supervised versus multiple instance learning: an empirical comparison," in Proceedings of the 22nd International Conference on Machine Learning (ICML'05), Bonn, Germany, 2005, pp. 697-704. http://dx.doi.org/10.1145/1102351.1102439

[29] Y. Chen, J. Bi, and J. Z. Wang, "MILES: multiple-instance learning via embedded instance selection," IEEE Transactions on Pattern Analysis and Machine Intelligence, vol. 28, no. 12, pp. 1931-1947, 2006. http://dx.doi.org/10. 1109/tpami.2006.248

[30] C. Yang, M. Dong, and F. Fotouhi, "Region based image annotation through multiple-instance learning," in Proceedings of the 13th Annual ACM International Conference on Multimedia, Singapore, 2005, pp. 435-438. http://dx.doi.org/10.1145/1101149.1101245

[31] B. Babenko, M. H. Yang, and S. Belongie, "Robust object tracking with online multiple instance learning," IEEE Transactions on Pattern Analysis and Machine Intelligence, vol. 33, no. 8, pp. 1619-1632, 2011. http: //dx.doi.org/10.1109/tpami.2010.226

[32] P. L. Lanzi, W. Stolzmann, and S. W. Wilson, Learning Classifier Systems: From Foundations to Applications (LNCS vol.1813). Berlin: Springer, 2000.

[33] L. A. Zadeh, "Fuzzy sets," Information and Control, vol. 8, no. 3, pp. 338-353, 1965. http://dx.doi.org/10.1016/S00199958(65)90241-X

[34] E. H. Mamdani and S. Assilian, "An experiment in linguistic synthesis with a fuzzy logic controller," International Journal of Human-Computer Studies, vol. 51, no. 2, pp. 135-147, 1999. http://dx.doi.org/10.1006/ijhc.1973.0303

[35] E. H. Mamdani, "Application of fuzzy logic to approximate reasoning using linguistic synthesis," IEEE Transactions on Computers, vol. 26, no. 12, pp. 1182-1191, 1977. http://dx.doi.org/10.1109/tc.1977.1674779

[36] T. Takagi and M. Sugeno, "Fuzzy identification of systems and its applications to modeling and control," IEEE Transactions on Systems, Man and Cybernetics, vol. 15, no. 1, pp. 116-132, 1985. http://dx.doi.org/10.1109/tsmc. 1985.6313399 
[37] R. R. Yager, "On the theory of bags," International Journal of General Systems, vol. 13, no. 1, pp. 23-37, 1986. http://dx.doi.org/10.1080/03081078608934952

[38] K. T. Atanassov, "Intuitionistic fuzzy sets," Fuzzy Sets and Systems, vol. 20, no. 1, pp. 87-96, 1986. http://dx.doi. org/10.1016/s0165-0114(86)80034-3

[39] V. Torra, "Hesitant fuzzy sets," International Journal of Intelligent Systems, vol. 25, no. 6, pp. 529-539, 2010. http://dx.doi.org/10.1002/int.20418

[40] D. Ramot, R. Milo, M. Friedman, and A. Kandel, "Complex fuzzy sets," IEEE Transactions on Fuzzy Systems, vol. 10, no. 2, pp. 171-186, 2002. http://dx.doi.org/10.1109/91. 995119

[41] G. Cheng and J. Yang, "Complex fuzzy reasoning schemes," in Proceedings of 2010 3rd International Conference on Information and Computing (ICIC), Wuxi, China, 2010, pp. 29-32. http://dx.doi.org/10.1109/icic. 2010.190

[42] V. G. Kaburlasos and A. Kehagias, "Fuzzy inference system (FIS) extensions based on the lattice theory," IEEE Transactions on Fuzzy Systems, vol. 2, no. 3, pp. 531-546, 2013. http://dx.doi.org/10.1109/TFUZZ.2013.2263807

[43] A. Mahnot and M. Popescu, "Fumil-fuzzy multiple instance learning for early illness recognition in older adults," in Proceedings of 2012 IEEE International Conference on Fuzzy Systems (FUZZ-IEEE), Brisbane, Australia, 2012, pp. 1-5. http://dx.doi.org/10.1109/fuzz-ieee.2012. 6251358

[44] J. Casasnovas and G. Mayor, "Discrete t-norms and operations on extended multisets," Fuzzy Sets and Systems, vol. 159, no. 10, pp. 1165-1177, 2008. http://dx.doi.org/ 10.1016/j.fss.2007.12.005

[45] D. E. Tamir and A. Kandel, "Axiomatic theory of complex fuzzy logic and complex fuzzy classes," International Journal of Computers, Communications \& Control, vol. 6, no. 3, pp. 562-576, 2011. http://dx.doi.org/10.15837/ijccc. 2011.3.2135

[46] J. S. R. Jang, C. T. Sun, and E. Mizutani, Neuro-fuzzy and Soft Computing: A Computational Approach to Learning and Machine Intelligence. Upper Saddle River, NJ: Prentice Hall, 1997.
[47] D. Dubois and H. M. Prade, Fuzzy Sets and Systems: Theory and Applications. New York, NY: Academic Press, 1980.

[48] M. Xia and Z. Xu, "Hesitant fuzzy information aggregation in decision making," International Journal of Approximate Reasoning, vol. 52, no. 3, pp. 395-407, 2011. http://dx.doi.org/10.1016/j.ijar.2010.09.002

[49] L. A. Zadeh, "Is there a need for fuzzy logic?," Information Sciences, vol. 178, no. 13, pp. 2751-2779, 2008. http://dx.doi.org/10.1016/j.ins.2008.02.012

[50] A. Karem and H. Frigui, "Fuzzy clustering algorithm of multiple instance data," in Proceedings of 2015 IEEE International Conference on Fuzzy Systems (FUZZ-IEEE), Istanbul, Turkey, 2015, pp. 1-7. http://dx.doi.org/10.1109/ FUZZ-IEEE.2015.7338084

[51] J. C. Bezdek, Pattern Recognition with Fuzzy Objective Function Algorithms. New York, NY: Plenum Press, 1981.

[52] H. Frigui and P. Gader, "Detection and discrimination of land mines in ground-penetrating radar based on edge histogram descriptors and a possibilistic k-nearest neighbor classifier,' IEEE Transactions on Fuzzy Systems, vol. 17, no. 1, pp. 185-199, 2009. http://dx.doi.org/10.1109/tfuzz. 2008.2005249

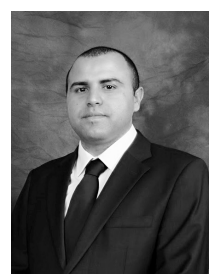

Amine B. Khalifa received the Ph.D. degree in computer science and engineering from the University of Louisville, Louisville, KY in 2015. Prior to that, he received a B.S. degree in telecommunication engineering in 2009 from Tunisia Higher School of Communication (SUPCOM), Tunis, His research interests are in the area of fuzzy inference, machine learning, and computer vision.

E-mail: amine.benkhalifa@louisville.edu

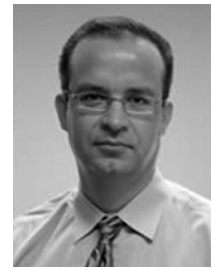

Hichem Frigui is a Professor of Computer Engineering and Computer Science and Director of the Multimedia Research Lab at the University of Louisville, KY. He received the $\mathrm{Ph} . \mathrm{D}$. degree in computer engineering and computer science from the University of Missouri, Columbia, in 1997. From 1998 to 2004, he was an Assistant Professor with The University of Memphis, Memphis, TN. He has been active in the research fields of fuzzy pattern recognition, data mining, and image processing with applications 
to content-based multimedia retrieval and land mine detection.

He served as an Associate Editor of the IEEE Transactions on Fuzzy Systems and Fuzzy Sets and Systems. He was a member of few teams that developed, implemented, and field-tested several real-time algorithms for mine detection using ground penetrating radar sensors. His research has been funded by the National Science Foundation (NSF), Office of Naval Research (ONR), Army Research Office (ARO), and Kentucky Science and Engineering Foundation (KSEF). He has coauthored over 170 technical publications. In 2002, he has received the $\mathrm{Na}-$ tional Science Foundation Career Award for outstanding young scientists. His research interests are in the area of fuzzy pattern recognition, data mining, content-based multimedia retrieval, and land mine detection.

E-mail: h.frigui@louisville.edu 